\title{
Estenose traqueal pós-ventilação mecânica em pacientes acometidos pela Covid-19: evento isolado ou complicação direta?
}

\author{
Tracheal stenosis post mechanical ventilation in patients affected by Covid-19: isolated \\ event or direct complication?
}

Estenosis traqueal post ventilación mecánica en pacientes afectados por Covid-19: ¿evento aislado o complicación directa?

Pedro Duarte Moreira Andrade ${ }^{1 *}$, Ana Luiza Faria Rabelo ${ }^{1}$, Camila Baquieti Carminate ${ }^{1}$, Camilla de Freitas Maziero', Gabriel Calafange Cunha ${ }^{1}$, Ian Martins da Silveira ${ }^{2}$, Larissa Mariani Rezende Almeida $^{1}$, Layandra Vittória de Assis ${ }^{1}$, Silvia Aparecida de Oliveira Nascimento Silva ${ }^{1}$, Layara de Assis ${ }^{1}$.

\section{RESUMO}

Objetivo: Identificar se a origem da estenose laringotraqueal pós-intubação (PILS) é uma complicação direta da covid-19 ou se acontece em pacientes em eventos isolados/raros. Revisão bibliográfica: Os resultados apresentados na literatura demonstram que existe a associação direta da PILS em pacientes com COVID-19, principalmente naqueles que ficam um longo período em intubação orotraqueal (IOT) e nos que possuem alguma comorbidade prévia, como Hipertensão Arterial Sistêmica, Diabetes, Asma ou alguma outra síndrome respiratória. Considerações finais: Este estudo demonstrou que a estenose de traqueia pós-intubação é uma complicação menos comum, mas que ocorre, em pacientes com COVID-19, principalmente nos susceptíveis e que ficam mais de 3 semanas em ventilação mecânica. Além disso, um bom trabalho em equipe nas unidades de terapia intensiva (UTIs), entre os clínicos gerais, médicos de pronto-socorro e outros profissionais médicos é fundamental para direcionar esses pacientes aos cuidados de um centro terciário com experiência adequada no campo da cirurgia das vias aéreas, melhorando o prognóstico do paciente.

Palavras-chave: Covid-19, Estenose de traqueia, Ventilação mecânica.

\begin{abstract}
Objective: To identify whether the origin of post-intubation laryngotracheal stenosis (PILS) is a direct complication of covid-19 or if it happens in patients with isolated/rare events. Literature review: The results presented in the literature demonstrate that there is a direct association of PILS in patients with COVID-19, especially in those who spend a long period in orotracheal intubation (OTI) and in those who have some prior comorbidity, such as Systemic Arterial Hypertension, Diabetes, Asthma or some other respiratory syndrome. Final considerations: This study demonstrated that post-intubation tracheal stenosis is a less common complication, but that it occurs in patients with COVID-19, especially in susceptible patients who spend more than 3 weeks on mechanical ventilation. In addition, good teamwork in intensive care units (ICUs) among general practitioners, emergency room physicians and other medical professionals is essential to direct these patients to the care of a tertiary center with adequate experience in the field of surgery. airway, improving the patient's prognosis.
\end{abstract}

Key words: Covid-19, Tracheal stenosis, Mechanical ventilation.

\footnotetext{
${ }^{1}$ Instituto Metropolitano de Ensino Superior (IMES UNIVAÇO), Ipatinga - MG.

*E-mail: pedroduarte08@hotmail.com

2 Universidade Federal do Vale do São Francisco (UNIVASF), Petrolina - PE.
} 


\section{RESUMEN}

Objetivo: Identificar si el origen de la estenosis laringotraqueal postintubación (PILS) es una complicación directa del covid-19 o si ocurre en pacientes con eventos aislados / raros. Revisión bibliográfica: Los resultados presentados en la literatura demuestran que existe una asociación directa de PILS en pacientes con COVID-19, especialmente en aquellos que pasan un período prolongado en intubación orotraqueal (OTI) y en aquellos que tienen alguna comorbilidad previa, como Hipertensión arterial sistémica, diabetes, asma o algún otro síndrome respiratorio. Consideraciones finales: Este estudio demostró que la estenosis traqueal posterior a la intubación es una complicación menos común, pero que ocurre en pacientes con COVID-19, especialmente en pacientes susceptibles que pasan más de 3 semanas con ventilación mecánica. Además, el buen trabajo en equipo en las unidades de cuidados intensivos ( $\mathrm{UCl}$ ) entre médicos generales, médicos de urgencias y otros profesionales médicos es fundamental para dirigir a estos pacientes a la atención de un centro terciario con adecuada experiencia en el campo de la cirugía. pronóstico.

Palabras clave: Covid-19, Estenosis traqueal, Ventilación mecánica.

\section{INTRODUÇÃO}

Em dezembro de 2019, iniciaram-se os relatos dos primeiros casos de infecção pelo novo coronavírus (SARS-coV-2) na cidade de Wuhan, na China. A humanidade ficou atenta ao que posteriormente viria a se tornar, segundo a Organização Mundial da Saúde (OMS), uma Emergência de Saúde Pública de Nível Internacional. Dessa forma, houve um expressivo aumento no número de ocorrências concomitantemente a disseminação intercontinental da doença e poucos meses depois a COVID-19 foi tipificada como uma pandemia (WU T, et al., 2020).

De acordo com Mitra RL, et al. (2020), paralelo ao alastramento da enfermidade, sobreveio o crescimento da morbidade e mortalidade. Ademais, a aplicação de indicadores do "Pandemic Severity Assessment Framework" (PSAF) mostra uma doença altamente transmissível, do mesmo modo os indicadores de gravidade clínica sugerem alta gravidade. Por fim, chegando a atingir em julho de 2021, uma marca de 184 milhões de casos e 4 milhões de mortes em todo o mundo (WORLD HEALTH ORGANIZATION, 2021).

Na maioria dos pacientes, a doença assume uma forma leve com sintomas como febre, tosse, náusea, vômito e diarreia, mas também pode causar envolvimento maciço do trato respiratório inferior com pneumonia intersticial (GUAN WJ, et al., 2020). Apesar da baixa taxa de mortalidade (1,4-2,3\%) e do número relativamente baixo de pacientes que precisam de cuidados intensivos (5 a 12\%), o SARS-CoV-2 está colocando uma grande carga nos sistemas de saúde em todo o mundo, causando uma sobrecarga sem precedentes nas instalações hospitalares, especialmente nas unidades de terapia intensiva (UTI), devido à sua alta transmissibilidade e ao grande número de pacientes infectado (KOWALSKI LP, et al., 2020).

Na experiência recente, o tempo médio desde o início dos sintomas até o desenvolvimento de pneumonia foi de aproximadamente 5 dias, enquanto o tempo médio desde o início dos sintomas até a admissão na UTI por hipoxemia grave foi de aproximadamente 7-12 dias. A causa da hipoxemia grave foi essencialmente correlacionada à síndrome do desconforto respiratório agudo em $60-70 \%$ dos casos, seguida por choque em 30\%, disfunção miocárdica em 20-30\% e insuficiência renal aguda em 10-30\% (PHUA J, et al., 2020).

Pacientes com COVID-19 admitidos na UTI geralmente requerem ventilação mecânica prolongada com alta Pressão Expiratória Final Positiva (PEEP) por meio de um tubo endotraqueal (TAY JK, et al., 2020). Em configurações pré-COVID-19, a traqueostomia realizada após 7-14 dias da intubação endotraqueal melhorou significativamente a chance de desmame bem-sucedido e reduziu o risco de complicações e mortalidade quando comparada à manutenção a longo prazo do tubo orotraqueal no local. No entanto, a prática clínica atual para pacientes com COVID-19 admitidos em UTIs em todo o mundo é tentar adiar ao máximo a traqueostomia até que o paciente não precise mais de ventilação mecânica e esteja livre do vírus (MATTIOLI F, et al., 2020). 
Isso se deve principalmente ao alto risco de decanulação acidental durante a ventilação mecânica e à chance de infecção cruzada de profissionais de saúde devido à duração prolongada da positividade viral traqueal em comparação com a carga viral salivar (SOMMER DD, et al., 2020). Infelizmente, quando estritamente seguida, essa política pode significar que os pacientes permanecem intubados por até 3-4 semanas, o que aumenta consideravelmente lesões à mucosa e necrose da parede traqueal, levando a uma estenose laringotraqueal após intubação (SOMMER DD, et al., 2020).

A taxa de estenose laringotraqueal pós-intubação (PILS) em pacientes não COVID-19 é de 10-22\%, sendo uma condição rara com uma incidência estimada de 4,9 casos por milhão por ano na população em geral (MIWA M, et al., 2021). A PILS torna-se sintomática quando a estenose inclui> $60 \%$ do lúmen traqueal. Relatórios anteriores mostraram que o tempo de início da PILS variou de 28 dias a 6 meses após a extubação, sendo a causa mais comum iatrogênica, especificamente em intubações prolongadas, hiperinsuflação do balonete do tubo endotraqueal e uso de tubos grandes. Outros fatores de risco incluem infecções peritubulares, idade avançada, efeito feminino e do estrogênio, tabagismo, obesidade e diabetes (GERVASIO CF, et al., 2020).

Ainda não há dados sobre a ocorrência de PILS em pacientes com COVID-19 recuperados, no entanto, acredita-se que esses pacientes possam ter maior risco de desenvolver PILS que os pacientes que não têm covid, devido ao seu estado hiperinflamatório, que causa fibrose alterada, mas também porque esses pacientes são submetidos a intubações prolongadas e geralmente já apresentam comorbidades, como diabetes e hipertensão (GERVASIO CF, et al., 2020).

Considerando estes aspectos, o presente estudo teve como objetivo identificar se a origem da PILS é uma complicação direta da covid-19 ou se acontece em pacientes como eventos raros.

\section{REVISÃO BIBLIOGRÁFICA}

A idade média foi de 68,8 anos no grupo COVID-19 e 68 no grupo de controle, sendo oito (27\%) e 15 (33\%) mulheres que estavam inscritas no grupo COVID-19 e no grupo controle, respectivamente (PRINCE ADP, et al., 2020). Entre 98 pacientes consecutivos com COVID-19 com insuficiência respiratória grave admitidos em 2020, 30 foram submetidos à VM invasiva prolongada. Quarenta e cinco controles consecutivos pacientes pareados por idade e sexo foram selecionados de acordo com critérios de inclusão e exclusão no tempo (SICILIANI A, et al., 2020).

Quatorze pacientes (47\%) no grupo COVID-19 tinha FTTLs $(n=10,33 \%)$ ou TEFs ( $n=4,13 \%)$, enquanto 1 paciente (2\%) no grupo de controle tinha FTTLs $(\mathrm{OR}, 38,4,95 \% \mathrm{Cl}, 4,7-316,9)$. Este último teve uma ruptura do anel traqueal diagnosticada durante o procedimento de traqueostomia (SCHNITTKER R, et al., 2020). Todos os 30 pacientes com COVID-19 foram submetidos a manobras de pronação e receberam terapia com esteróides, em comparação com $5(11 \%)$ e $14(31 \%)$ pacientes no grupo controle, respectivamente (SCHNITTKER R, et al., 2020).

Além disso, o grupo COVID-19 foi tratado com doses mais altas de esteroides (metil prednisolona intravenosa, $80 \mathrm{mg}$ vs $40 \mathrm{mg}$ ) e tinha pressão parcial de oxigênio arterial mais baixa para a fração de oxigênio inspirado ( $\mathrm{PaO} 2$ / FIO2) proporção durante a segunda semana de VM invasiva. Nenhuma outra diferença surgiu entre esses 2 grupos (GAO Q, et al., 2020).

Pacientes com COVID-19 com e sem complicações traqueais apresentavam características demográficas, comorbidades, parâmetros e duração da ventilação e manejo terapêutico semelhantes, sendo que dez (71\%) pacientes com dano de traqueia tiveram estenose de traqueia, 6 (43\%) tiveram pneumotórax e 13 (93\%) tiveram pneumomediastino, enquanto estas complicações não foram observadas em pacientes com COVID19 sem dano traqueal (GAO Q, et al., 2020).

Dentre os artigos analisados, todos os autores fizeram a associação da PILS em pacientes com COVID19 que passaram mais de 3 semanas intubados (TAY JK, et al., 2020). Aproximadamente $8 \%$ a $13 \%$ dos pacientes admitidos em unidades de terapia intensiva modernas e que requerem ventilação mecânica serão 
submetidos a traqueostomia (TAY JK, et al., 2020). A principal indicação para traqueostomia é a possibilidade de ventilação mecânica por um longo período de tempo sem possíveis complicações de uma longa duração IOT (SENTURK MR, et al., 2020).

A estenose pós-traqueotomia envolvendo a cartilagem cricóide geralmente é causada pela colocação elevada de uma traqueotomia com ruptura da cartilagem cricóide e do primeiro anel traqueal e infecção neste nível, sendo frequentes e apresentam um desafio no que diz respeito ao reparo (MCGRATH B, et al., 2020).

As razões para este número crescente de lesões das vias aéreas altas são incertas, mas podemos supor que a colocação de uma traqueotomia em cenário difícil (como um tratamento intensivo na unidade de cuidados dedicada ao COVID-19) ou inflamação das vias aéreas, como acontece em pacientes com COVID19, pode contribuir para a seu aumento adicional (MCGRATH B, et al., 2020).

É razoável prever que a ventilação com pressão positiva é necessária em pacientes com COVID-19 com consequente intubação e posterior traqueotomia juntamente com a inflamação das vias aéreas para fenômenos de vasculite que podem aumentar a taxa de lesões traqueais e originar estenose em um futuro próximo (PRINCE ADP, et al., 2020).

A pandemia da COVID-19 gerou um incrível desafio para o tratamento cirúrgico da estenose laringotraqueal porque 0 acesso às vias aéreas aberto durante cirurgia laringotraqueal cria uma situação de alto risco para cirurgiões, anestesiologistas e os assistentes sala de cirurgia (PRINCE ADP, et al., 2020). Embora alguns casos cirúrgicos de estenose laringotraqueal possam ser adiados, os pacientes com obstrução das vias aéreas ou sintomas progressivos, muitas vezes requerem intervenção cirúrgica urgente, consequentemente, medidas apropriadas devem ser tomadas para evitá-los e uma discussão sobre seu tratamento adequado e o tempo seria recomendado caso a caso (CARSANA L, et al., 2020).

Medidas preventivas para evitar estenose das vias aéreas devem ser empregadas. O manejo das vias aéreas, por intubação endotraqueal ou colocação de traqueostomia / cricotireotomia, deve ser reconhecido como um procedimento perigoso devido à potencial infecção cruzada transmitida pelo ar por pacientes infectados com o SARS-CoV-2 (COOK TM, et al., 2020).

Consequentemente, é de extrema importância que o clínico capacitado gerencie as vias aéreas para permitir seu estabelecimento bem-sucedido de maneira segura, precisa e rápida. O primeiro passo para isso é representado por um planejamento preciso, levando-se em consideração a possibilidade de difícil manejo das vias aéreas, possivelmente associada à laringite e edema laríngeo relacionados à COVID-19 (MCGRATH B, et al., 2020).

A intubação difícil é definida por tentativas repetidas de falha na introdução de um tubo endotraqueal, duração prolongada de tais manobras ou necessidade de múltiplas abordagens e / ou dispositivos de intubação, geralmente se refere aos Graus III ou IV da escala modificada de Cormack-Lehane (BARJAKTAREVIC I, et al., 2017). Somente a experiência do anestesiologista, após uma avaliação abrangente do paciente levando em consideração todos os dados disponíveis, pode prever uma intubação endotraqueal potencialmente difícil (SCHNITTKER R, et al., 2020).

Além disso, vários dispositivos como GlideScope ou endoscopia de fibra óptica transnasal estão agora disponíveis para ajudar no atendimento de emergência e médicos de UTI em uma situação tão difícil, certamente melhorando as chances de uma intubação bem-sucedida (COOK TM, et al., 2020). No entanto, algumas dessas ferramentas podem aumentar o risco de contaminação aérea, por esse motivo, em um cenário de pandemia COVID-19, diretrizes da British Difficult Airway Society, recomendam sempre usar Equipamento de Proteção Individual (EPI) adequado e usar o tubo com balonete maior possível (7,0-8,0 mm de diâmetro interno para mulheres e 8,0-9,0 mm para homens), inflando o manguito para selar as vias aéreas antes de iniciar a ventilação (COOK TM, et al., 2020).

Comum a todas as tentativas cirúrgicas e endoscópicas de criar uma via aérea artificial deve ser a intenção de entrar na traquéia no nível dos primeiros anéis, criando o mínimo de interrupção possível em termos de número de anéis danificados durante a abertura traqueal e posicionamento da cânula (MEHEL DM, et al., 2020). 
Deve ser inserida a menor cânula de traqueostomia possível, sempre levando em consideração o habitus corporal, morfologia do pescoço, e condições clínicas de um determinado paciente. Além disso, secreções espessas e a necessidade de broncoscopias frequentes para limpar as vias aéreas distais do tampão de muco podem inicialmente levar ao uso de cânulas maiores. Isso, no entanto, deve ser reduzido em tamanho assim que for seguro (DE KLEIJN BJ, et al., 2019).

Após a extubação ou decanulação do paciente, é necessário um período de janela de alguns dias antes de se considerar a estabilização definitiva das condições respiratórias do paciente. Normalmente, a PILS não se manifesta neste período de tempo, mas, sim, após a alta da UTI, frequentemente quando o paciente já está em casa, e não antes da terceira a quarta semana ou mais após o restabelecimento das condições respiratórias normais (DE KLEIJN BJ, et al., 2019).

Todos os pacientes com história de internação em UTI relacionada a COVID-19 devem ser acompanhados após a alta por um otorrinolaringologista ou outro especialista em vias aéreas para diagnosticar complicações precoces de forma proativa no nível da laringe e traquéia. Medicamentos sistêmicos e tópicos, desbridamento local, dilatações endoscópicas ou mesmo apenas vigilância de condições subclínicas podem ter papel fundamental para evitar que problemas importantes das vias aéreas como dispneia importante aos esforços e em repouso, variadamente associado a rouquidão, estridor, tosse seca e disfagia (SCHNITTKER R, et al., 2020).

Deve seguir-se o encaminhamento imediato dos pacientes para centros terciários com experiência específica em avaliação e tratamento adequado de PILS (SENTURK MR, et al., 2020). É de extrema importância tentar evitar, tanto quanto possível, que a estenose de traqueia pós-intubação seja diagnosticada erroneamente como asma ou outras condições pulmonares que causam dispneia, retardando assim o início dos processos diagnósticos e terapêuticos adequados (SENTURK MR, et al., 2020).

Nesse sentido, uma das ferramentas de diagnóstico de primeira linha mais comuns e úteis, também disponível em ambientes clínicos não especializados, é representada por testes de função pulmonar de rotina. Sua simplicidade e não invasividade são particularmente bem-vindas em uma triagem de PILS relacionada ao COVID-19, antes do encaminhamento a especialistas em vias aéreas para uma avaliação mais detalhada da condição subjacente (GERVASIO CF, et al., 2020).

Durante a pandemia, os tratamentos endoscópicos intervencionistas estão tendo um papel auxiliar no tratamento da estenose traqueal. A dilatação traqueal pode representar uma ponte para a cirurgia, ajudando a controlar com sucesso pacientes sintomáticos e retardando a cirurgia (BRINDLE EM et al., 2020). Nessa população, evitar o implante de stent traqueal é fundamental por causa de seu potencial dano ao tecido, exercendo fricção e pressão radial na parede das vias aéreas causando uma resposta inflamatória com granulação e estenoses adicionais que podem prejudicar o tratamento cirúrgico subsequente (BRINDLE EM et al., 2020).

Os procedimentos endoscópicos podem ser considerados como o primeiro tratamento em pacientes selecionados após a avaliação da estenose, como estenose não complexa com baixo grau de envolvimento da cartilagem ou traqueomalácia tendo uma taxa de sucesso de $60 \%$ após $1 \pm 3$ sessões (SICILIANI A, et al., 2020).

No que diz respeito às modalidades de traqueostomia, faltam ensaios clínicos prospectivos randomizados comparando diferentes técnicas cirúrgicas, como traqueostomia aberta, vs. vários tipos de abordagens percutâneas. No entanto, em um cenário COVID-19, a traqueostomia aberta parece representar riscos reduzidos para os pacientes infectados devido à entrada mais rápida das vias aéreas e menor aerossolização (TAY JK, et al., 2020).

Além do uso adequado de EPI, desde protetores faciais a respiradores purificadores de ar elétricos, o estabelecimento das vias aéreas cirúrgicas deve ser realizado à beira do leito para evitar o transporte desnecessário de pacientes da UTI para o centro cirúrgico e vice-versa. A equipe cirúrgica deve ser reduzida ao mínimo e o descarte de resíduos e / ou descontaminação dos equipamentos utilizados durante a cirurgia deve ser aplicado para minimizar o risco de contaminação ambiental (TAY JK, et al., 2020). 


\section{CONSIDERAÇÕES FINAIS}

Foi observado que a PILS é uma das complicações diretas da COVID-19, especialmente nos pacientes que ficam um tempo superior a 3 semanas com IOT. Assim, é altamente provável que nos próximos meses e anos os otorrinolaringologistas, bem como os cirurgiões torácicos, sejam chamados para tratar um número cada vez maior de estenose de traqueia pós intubação devido aos inúmeros problemas emergentes em todo o mundo relacionados à pandemia da COVID-19. Dessa maneira, o trabalho, além de demonstrar essa relação, contribuiu para orientar os profissionais da saúde, como os clínicos gerais, médicos de UTI a terem um bom trabalho em equipe para prevenir precocemente uma complicação das vias aéreas, impedindo a intubação do paciente, melhorando o seu prognóstico e evolução clínica.

\section{REFERÊNCIAS}

1. BARJAKTAREVIC I, et al. Ventilação não invasiva em difícil intubação endotraqueal: análise sistemática e de revisão. Anaesthesiol Intensive Ther, 2017; 49: 294-302.

2. BRINDLE EM, GAWANDE A. Gerenciando COVID-19 em sistemas cirúrgicos. Ann Surg, $2020 ; 272(1): 1-2$.

3. CARSANA L, et al. Pulmonary post-mortem findings in a series of COVID-19 cases from northern Italy: a two-centre descriptive study. Lancet Infect Dis, 2020.

4. COOK TM, et al. Diretrizes de consenso para o manejo das vias aéreas em pacientes com COVID-19. Anestesia, 2020; 75: 785-799.

5. DE KLEIJN BJ, et al. Complicações de curto e longo prazo de traqueotomias cirúrgicas e de dilatação percutânea: um grande estudo de coorte retrospectivo em um único centro. Eur Arch Otorhinolaryngol, 2019; 276: 1823-1828.

6. GAO Q, et al. The epidemiological characteristics of 2019 novel coronavirus diseases (COVID-19) in Jingmen, Hubei, China. MedRxiv, 2020.

7. GERVASIO CF, et al. Estenose traqueal após traqueostomia para ventilação mecânica em pneumonia COVID-19 relato de 2 casos do norte da Itália. O jornal americano de relatos de casos, 2020; 21.

8. GUAN WJ, et al. Características clínicas da doença por coronavírus em 2019 na China. N Engl J Med, 2020; 382 : 1708-1720.

9. KOWALSKI LP, et al. Pandemia de COVID-19: efeitos e recomendações baseadas em evidências para a prática de otorrinolaringologia e cirurgia de cabeça e pescoço. Pescoço, 2020.

10. MATTIOLI F, et al. Traqueostomia na pandemia de COVID-19. Eur Arch Otorhinolaryngol, 2020.

11. MCGRATH BA, et al. Tracheostomy in the COVID-19 era: global and multidisciplinary guidance Lancet Respir Med, 2020; 717-725.

12. MEHEL DM, et al. Classificação da lesão laríngea em pacientes com intubação prolongada e determinar os fatores que causam a lesão. Am J Otolaryngol, 2020.

13. MITRA RL, et al. "An algorithm for managing QT prolongation in coronavirus disease 2019 (COVID-19) patients treated with either chloroquine or hydroxychloroquine in conjunction with azithromycin: Possible benefits of intravenous lidocaine." HeartRhythm case reports, 2020; 6: 244-248

14. MIWA M, et al. Dois casos de estenose laringotraqueal pós-intubação ocorrendo após COVID-19 grave. Intern. Med, $2021 ; 60$ (3): 473-477.

15. PHUA J, et al. Manejo de terapia intensiva da doença coronavírus 2019 (COVID-19): desafios e recomendações. Lancet Respir Med, 2020; 8: 506-517.

16. PRINCE ADP, et al. Airway management for endoscopic laryngotracheal stenosis surgery during COVID-19. Otolaryngol Head Neck Surg, 2020; 78-80.

17. SCHNITTKER R, et al. Paciente e fatores cirúrgicos associados à incidência de falha e intubação difícil. Anestesia, 2020; 75: 756-766.

18. SICILIANI A, et al. Estado da arte em cirurgia traqueal: uma breve revisão da literatura. Multidiscip Respir Med, 2020; 13: 1-7.

19. SOMMER DD, et al. Recommendations from the CSO-HNS taskforce on performance of tracheotomy during the COVID-19 pandemic. J Otolaryngol Head Neck Surg, 2020;49(1):23.

20. ŞENTÜRK MR, et al.Thoracic anesthesia of patients with suspected or confirmed 2019 novel coronavirus infection: preliminary recommendations for airway management by the European Association of Cardiothoracic Anaesthesiology Thoracic Subspecialty Committee. J Cardiothorac Vasc Anesth, 2020; 2315-2327.

21. TAY JK, et al. Considerações cirúrgicas para traqueostomia durante a pandemia de COVID-19: lições aprendidas com o surto de Síndrome Respiratória Aguda Grave. JAMA Otolaryngol Head Neck Surg, 2020.

22. WORLD HEALTH ORGANIZATION. Coronavirus disease (COVID-19) pandemic: Numbers at a glance. 2021. Disponível em: https://covid19.who.int/. Acessado em: 10 de julho de 2021

23. WU T, et al. Controle do Intervalo QT para Prevenção de Torsades de Pointes Durante uso de Hidroxicloroquina e/ou Azitromicina em Pacientes com COVID 19. Arq. Bras. Cardiol, 2020; 114. 